\title{
MICROFLUIDIC DEVICES FOR RAPID LABEL-FREE SEPARATION OF CELLS AND POINT-OF-CARE DIAGNOSTICS

\author{
S. Bose ${ }^{l}$, M.-H. Hollatz ${ }^{l}$, C.-H. Lee ${ }^{l}$, J. M. Karp ${ }^{1,2}$ and R. Karnik ${ }^{l}$ \\ ${ }^{1}$ Massachusetts Institute of Technology, Cambridge, Massachusetts, USA \\ ${ }^{2}$ Harvard Medical School, Boston, Massachusetts, USA
}

\section{ABSTRACT}

Cell sorting serves an important role in clinical diagnosis and biological research. Most of the existing microscale sorting techniques are either non-specific to antigen type or rely on capturing cells making sample recovery difficult. We demonstrate a simple; yet effective technique for isolating cells in an antigen specific manner by using transient interactions of the cell surface antigens with asymmetric receptor patterned surface. Using microfluidic devices incorporating P-selectin patterns we demonstrate separation of HL60 cells from K562 cells. We achieved a sorting purity above $90 \%$ and efficiency greater than $85 \%$ with this system. We further demonstrate the clinical significance of the method by demonstrating single step separation of neutrophils from whole blood. When whole blood is introduced in the device, the granulocyte population gets separated exclusively yielding neutrophils of high purity $(<10 \%$ contamination). To our knowledge, this is the first ever demonstration of continuous label free sorting of neutrophils from whole blood.

\section{INTRODUCTION}

Separation and analysis of cells based on surface markers plays an important role in biological research and clinical diagnosis. Existing microscale cell sorting techniques are either non-specific to antigen type, require expensive accessory equipment for their operation, or capture cells on adhesive surfaces and necessitate special elution procedures for recovering the cells. We have recently demonstrated that transient receptor-ligand interactions that result in cell rolling [1] on a surface under fluid flow can be used to control the flow of cells on a receptorpatterned substrate [2], which paves the way for a new technique for label-free cell separation. Specifically, we found that when a rolling cell encounters a patterned P-selectin receptor edge, it is deflected from its direction of flow and follows the edge [2]. We envision a microfluidic device that would perform label-free separation of cells by rolling on receptor patterned surfaces (Figure $1)$.

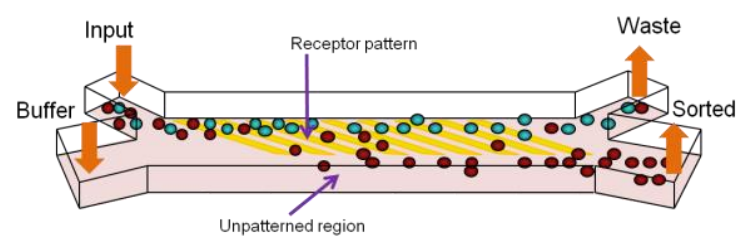

Figure 1: Schematic of the cell separation device. Cells are introduced from one side in a buffer flow. Rolling of cells on patterned receptors causes them to separate laterally from where they can be collected in different channels.

The technique relies on cell rolling - a physiological phenomenon exhibited by several types of cells including leukocytes, hematopoietic stem cells and cancer cells, which is mediated by glycoproteins known as selectins. In our earlier work we studied the dynamics of the rolling of HL60 cells on P-selectin pattern edges and described the optimal condition under which the deflection of cells on these asymmetric patterns can be maximized
[3]. In this work we report design of microfluidic devices for continuous-flow separation of i) HL60 cells from K562 cells and ii) granulocytes from whole blood, based on transient adhesive interactions between PSGL-1 expressed on the surface of HL60 cells and granulocytes with P-selectin receptors patterned in the device. P-selectin was patterned by selective surface chemistry on gold-patterned glass slides. The design allows target cells to settle gravitationally along the length of the channel, after which the cells get displaced laterally into a non-patterned region for quick elution.

\section{EXPERIMENTAL}

Gold coated glass slides (EMF corp.) were patterned using a positive photoresist as an etching mask. The substrates were cleaned with piranha $(3: 1, \mathrm{H} 2 \mathrm{SO} 4: \mathrm{H} 2 \mathrm{O} 2)$ and immersed in $1 \%$ PEG-trimethoxysilane (Gelest) solution in toluene overnight followed by treatment with $1 \mathrm{mM}$ dithio-bis-succinimidyl propionate (DSP, Pierce Biotech.) in DMF for $2 \mathrm{~h}$, after which they were washed in ethanol. Finally the substrates were incubated with P-selectin (R\&D Systems) and stored in 1\% BSA solution until used in the experiments (Figure 2).

The flow cell was molded in PDMS using standard lithography techniques, and consisted of a serpentine channel of rectangular cross-section (100 $\mathrm{m} \mathrm{x} 1 \mathrm{~mm})$ with total length of 20 $\mathrm{cm}$. The device was attached to the substrate after alignment by applying vacuum, creating a reversible bond.

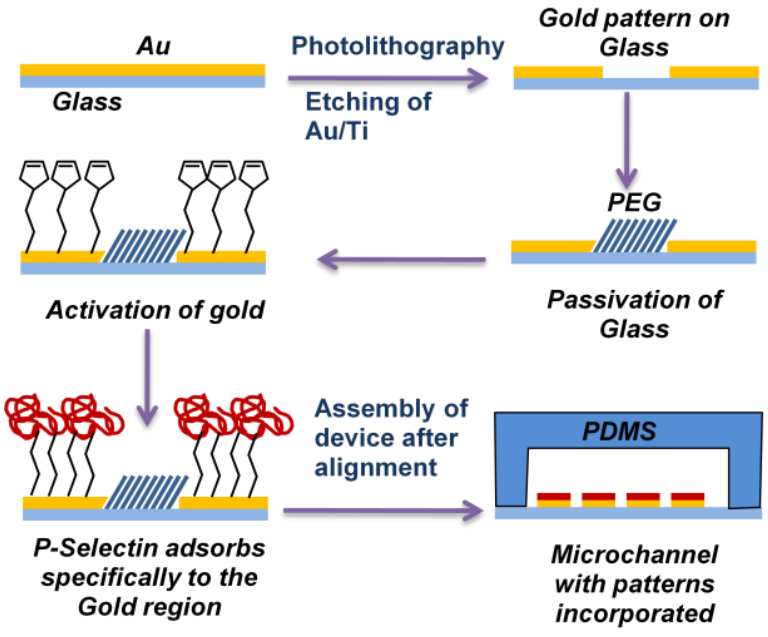

Figure 2: Fabrication process to create receptor pattered substrate through selective chemistry followed by alignment of device.

HL60 cells and K562 cells (ATCC) stained with CellTracker Red and Green respectively, were mixed in 1:1 ratio with a total concentration of 1 million cells per $\mathrm{mL}$, and injected into the device assembly alongside a buffer stream. Flux of the flowing cells was recorded at different locations along the channel length using an epifluorescence microscope. For the separation of granulocytes, anticoagulated human whole blood was used instead of the cell mixture. The samples were analyzed via flow cytometry. 


\section{RESULTS AND DISCUSSION}

When a stream of HL60 and K562 cell mixture was introduced parallel to a buffer stream in 1:9 ratio at a wall shear stress of $0.5 \mathrm{dyn} / \mathrm{cm}^{2}$, we observed that HL60 cells specifically interacted with the patterns, followed the edges of the patterns, and were displaced laterally in a device length-dependent manner (Figure 3a). In contrast, the K562 cells showed a passive spreading across the width of the channel, possibly due to cell-cell hydrodynamic interactions. As evident from Figure $3 \mathrm{a}$, the efficiency and purity of the separation can be tailored depending on the fraction of the flow isolated at the channel exit. Collecting $25 \%$ of the flow from the purified end resulted in a purity of $94 \%$ of the separated HL60 population and a recovery efficiency of $\sim 85 \%$ (Figure $3 b$ ).
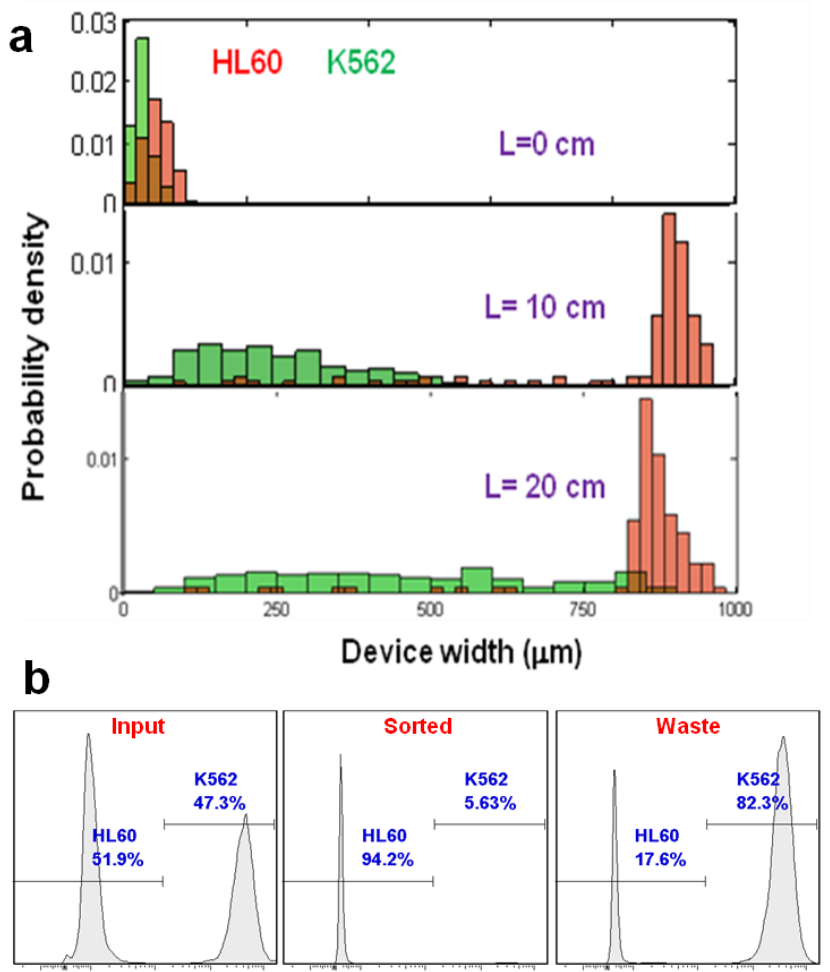

Figure 3: Separation of HL60 cells from K562 cells. (a) Distribution of the flux of HL60 and K562 cells evolving along the length of device. (b) Analysis of the input, sorted and waste samples via flow cytometry.

Next, we infused anticoagulated whole blood into the device and observed that similar to HL60 cells, neutrophils specifically attached and rolled on the P-selectin patterns getting displaced. The composition of the input and sorted sample was found by staining the samples using a pan leukocyte marker (CD45) and granulocyte specific marker (CD66). Amazingly, we found that the sorted sample was highly enriched in granulocytes $(>90 \%)$ as compared to $\sim 47 \%$ in the input (Figure 4 ), and significantly depleted of RBCs. We operated these devices for more than $3 \mathrm{~h}$ and did not find appreciable difference in performance indicating that the surfaces are resistant to bio-fouling.
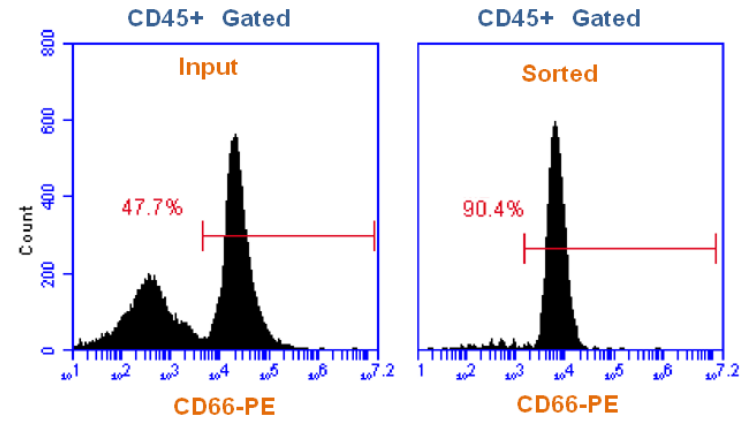

Figure 4: Separation of granulocytes from whole blood. The granulocyte composition of the input and sorted samples were analyzed via flow cytometry after staining by CD45-FITC (pan leukocyte marker) and CD66-PE (granulocyte marker) and are shown in the figure.

\section{CONCLUSION}

In this paper we demonstrated high efficiency, high purity separation of HL60 cells from K562 cells and granulocytes from whole blood by rolling on surfaces patterned with P-selectin in a continuous flow. Further development of this technology could lead to separation and detection of different cell types based on their differential rolling behavior on receptor edges. Although Pselectin was used in this study, other molecules that enable transient cell-surface interactions can potentially be used to target cells based on surface markers. This approach to continuous-flow separation of cells based on surface markers is promising for diagnostic applications and also as a general cell sorting technique.

\section{ACKNOWLEDGEMENTS}

The authors acknowledge the funding from the Deshpande Center for Technological Innovation at MIT (R.K. and J.M.K.), NIH grants HL-095722 and HL-097172 (J.M.K.) and NSF CAREER award 0952493 through the Chemical and Biological Separations program (R.K.) . The devices were fabricated at the Microsystems Technology Laboratory at MIT.

\section{REFERENCES}

[1] S. Q. Chen, R. Alon, R. C. Fuhlbrigge, and T. A. Springer, “ Rolling and transient tethering of leukocytes on antibodies reveal specializations of selectins “, PNAS, pp. 3172, vol 98, 1997.

[2] R. Karnik, S. Hong, H, Zang, Y. Mei, D. Anderson, J. Karp and R. Langer, "Nanomechanical control of cell rolling in two dimensions through surface patterning of receptors", Nano Letters., pp. 1153, vol 8, 2008.

[3] C-H Lee et.al., "Examining the lateral displacement of HL60 cells rolling on asymmetric P-selectin patterns", Langmuir, pp. 240, vol 27(1), 2011.

\section{CONTACT}

*R. Karnik, tel: +1-617-324-1155; karnik@mit.edu 